University of Nebraska - Lincoln

DigitalCommons@University of Nebraska - Lincoln

2009

\title{
Surface-Initiated Titanium-Mediated Coordination Polymerization from Catalyst-Functionalized Single and Multiwalled Carbon Nanotubes
}

Dimitrios Priftis

Nikolaos Petzetakis

Georgios Sakellariou

Marinos Pitsikalis

Durairaj Baskaran

See next page for additional authors

Follow this and additional works at: https://digitalcommons.unl.edu/usdoepub

Part of the Bioresource and Agricultural Engineering Commons

Priftis, Dimitrios; Petzetakis, Nikolaos; Sakellariou, Georgios; Pitsikalis, Marinos; Baskaran, Durairaj; Mays, Jimmy W.; and Hadjichristidis, Nikos, "Surface-Initiated Titanium-Mediated Coordination Polymerization from Catalyst-Functionalized Single and Multiwalled Carbon Nanotubes" (2009). US Department of Energy Publications. 68.

https://digitalcommons.unl.edu/usdoepub/68

This Article is brought to you for free and open access by the U.S. Department of Energy at DigitalCommons@University of Nebraska - Lincoln. It has been accepted for inclusion in US Department of Energy Publications by an authorized administrator of DigitalCommons@University of Nebraska - Lincoln. 


\section{Authors}

Dimitrios Priftis, Nikolaos Petzetakis, Georgios Sakellariou, Marinos Pitsikalis, Durairaj Baskaran, Jimmy W. Mays, and Nikos Hadjichristidis 


\title{
Surface-Initiated Titanium-Mediated Coordination Polymerization from Catalyst-Functionalized Single and Multiwalled Carbon Nanotubes
}

\author{
Dimitrios Priftis, ${ }^{\dagger}$ Nikolaos Petzetakis, ${ }^{\dagger}$ Georgios Sakellariou, ${ }^{\dagger} * *$ Marinos Pitsikalis, ${ }^{\dagger}$ \\ Durairaj Baskaran, ${ }^{\S}$ Jimmy W. Mays, ${ }^{*, \S}$ and Nikos Hadjichristidis*, ${ }^{\dagger}$
}

\author{
Department of Chemistry, University of Athens, 15771 Panepistimiopolis Zografou, Athens, Greece, \\ Department of Chemistry, University of Tennessee, 552 Buehler Hall, Knoxville, Tennessee 37996, and \\ Chemical Sciences Division and Center for Nanophase Materials Sciences, Oak Ridge National \\ Laboratory, Oak Ridge, Tennessee 37831
}

Received December 9, 2008; Revised Manuscript Received March 9, 2009

\begin{abstract}
Single (SWNTs) and multiwalled (MWNTs) carbon nanotubes were functionalized with a titanium alkoxide catalyst through a Diels-Alder cycloaddition reaction. The catalyst-functionalized carbon nanotubes (CNTs) were used for the surface initiated titanium-mediated coordination polymerizations of L-lactide (L-LA), $\epsilon$-caprolactone $\left(\epsilon\right.$-CL) and $n$-hexyl isocyanate (HIC) employing the "grafting from" technique. ${ }^{1} \mathrm{H}$ NMR, IR and Raman spectra showed that the precursor catalyst was successfully synthesized and covalently attached on the CNTs surface. Thermogravimetric analysis (TGA) revealed that the grafted poly(L-lactide) (PLLA) content could be controlled with time. The final polymer-grafted CNTs were readily dissolved in organic solvents as compared to the insoluble pristine and catalyst-functionalized CNTs. The presence of thick layers of polymers around the CNTs was observed through transmission electron microscopy (TEM). Differential scanning calorimetry (DSC) proved that the glass transition $\left(T_{\mathrm{g}}\right)$ and melting $\left(T_{\mathrm{m}}\right)$ temperatures of the PLLA are affected by the presence of the CNTs, while PLLA $\alpha$-helix conformation remains intact, as revealed by the circular dichroism (CD) spectra.
\end{abstract}

\section{Introduction}

Since the unique structure and the outstanding properties of carbon nanotubes (CNTs) were discovered, many researchers have focused their attention on potential applications, such as field emission displays, ${ }^{1}$ sensors ${ }^{2}$ and biological/biomedical systems and devices. ${ }^{3}$ CNTs have the tendency to exist in bundles rather than as individual tubes, because of strong van der Waals interactions, leading to insolubility in most organic media, and therefore limiting the range of applications. The dissolution of CNTs in various organic and aqueous solvents can however be greatly improved through the chemical attachment of macromolecules. Polymer-grafted CNTs combine the physicochemical properties of polymers and the extraordinary properties of the CNTs, such as mechanical strength and electrical conductivity. ${ }^{4}$

We have been engaged in the development of such materials for the covalent functionalization of CNTs, through the "grafting to" and "grafting from" techniques. The first approach ${ }^{5-7}$ generally takes advantage of the carboxylic acid groups, created by oxidation of the CNTs, followed by amidation or esterification with amine- or hydroxyl-terminated polymers. However, the availability of the carboxylic acid groups in the defects and the tips of the CNTs plus the steric hindrance encountered during the attachment of macromolecules lead to a low degree of grafting. ${ }^{8}$ In addition, the grafting of end-functionalized polymer chains is complicated by their adsorption and wrapping. ${ }^{6-8} \mathrm{On}$ the other hand, the "grafting from" technique ${ }^{9-12}$ involves the immobilization of initiator molecules on the CNT surface. In this way, high grafting density can be achieved and a variety of monomers can be polymerized from the CNT surface.

\footnotetext{
* Corresponding authors. E-mail: (N.H.) hadjichristidis@chem.uoa.gr or (G.S.) gsakellariou@chem.uoa.gr.

Department of Chemistry, University of Athens.

\$Chemical Sciences Division and Center for Nanophase Materials Sciences, Oak Ridge National Laboratory.

${ }^{\S}$ Department of Chemistry, University of Tennessee.
}

Functionalization $^{13,14}$ of CNTs with well-defined polymers has been described using several living or controlled polymerization methods including anionic, ${ }^{15,16}$ atom transfer radical polymerization (ATRP), ${ }^{9}$ reversible addition-fragmentation transfer (RAFT), ${ }^{17,18}$ and nitroxide mediated radical polymerization (NMRP) ${ }^{19}$ using either of the two grafting techniques. Recently, a few groups have investigated the CNT surface initiated ring-opening ${ }^{20,21}$ (ROP) and ring-opening metathesis polymerization $^{22}$ (ROMP). These methods are important since the metal catalysts are tolerant to polar, apolar and charged functional groups. Gomez et al. ${ }^{23}$ grafted a ruthenium alkylidene catalyst to the surface of SWNTs and subsequently used it to polymerize norbornene.

Poly(L-lactide), PLLA, and poly( $\epsilon$-caprolactone), PCL, are among the few biocompatible and biodegradable polymeric materials ${ }^{24}$ currently used in the biomedical field (e.g., sutures, drug delivery, etc. $)^{25}$ and therefore many attempts have been made to attach them to nanomaterials, such as CNTs. Yang et al. ${ }^{26}$ used a 6-arm star PLLA with a condensed triphenylbenzene core to interact with the surface of CNTs $(\pi-\pi$ interactions), while Olalde et al. ${ }^{27}$ used an oxidation/esterification process to functionalize single and multiwalled carbon nanotubes with PLLA. For grafting PCL and PLLA, the most commonly used catalyst is $\mathrm{Sn}(\mathrm{Oct})_{2}$. Zeng et al. ${ }^{20}$ and Buffa et al. ${ }^{21}$ have used $\mathrm{Sn}(\mathrm{Oct})_{2}$ to grow PCL chains from CNTs while Chen et al. ${ }^{28}$ have used it for the synthesis of PLLA functionalized MWNTs. In situ polycondensation ${ }^{29}$ of L-lactic acid with MWNTs is another technique that has been employed for the same purpose. However, in all these cases, the degree of polymer grafting in the final nanocomposite material did not exceed $60 \mathrm{wt} \%$, even for long reaction times $(20 \mathrm{~h})$.

Since the discovery of the first Ziegler-Natta catalytic system, titanium complexes have been extensively used for the synthesis of polymers with various macromolecular architectures. Novak was the first to use titanium complexes to control molecular weight and to produce relatively narrow molecular weight distribution polyisocyanates. ${ }^{30}$ Titanocenes and half- 
Scheme 1. Grignard Synthesis of (1-Benzocyclobutene ethoxy)dichlorocyclopentadienyltitanium (BCB-EOTiCpCl (1) $_{2}$ and Covalent Functionalization of MWNTs Using a [4 + 2] Diels-Alder Cycloaddition Reaction (2)
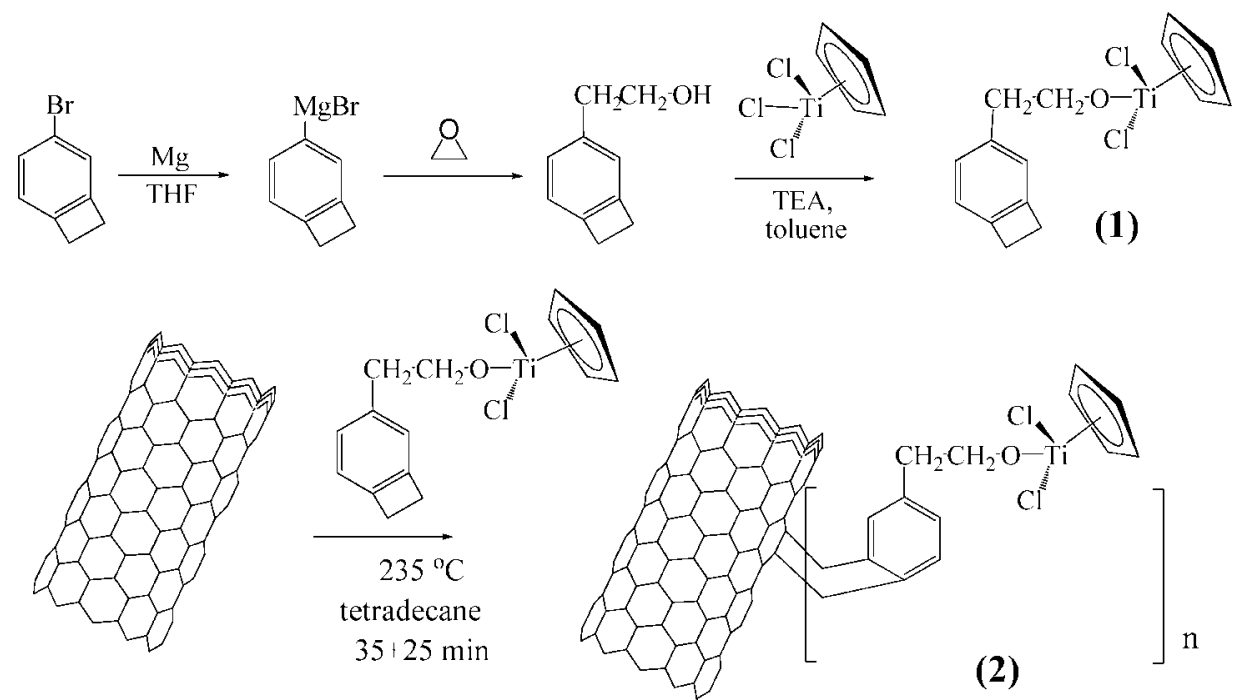

titanocenes have also been used for the polymerization of higher $\alpha$-olefins, ${ }^{31}$ dienes, ${ }^{32}$ styrene, ${ }^{33} n$-hexyl isocyanate (HIC),${ }^{34}$ $\epsilon$-CL, ${ }^{24,35}$ and methacrylates. ${ }^{36}$ Even though it is clear that titanium-based catalytic systems can be used for the synthesis of a broad variety of polymers, only a few attempts have been made for grafting CNT surfaces. Tong et al. ${ }^{37}$ used a titanium catalyst for the in situ Ziegler-Natta polymerization of ethylene from SWNTs. The functionalized SWNTs were mixed with commercial poly(ethylene) (PE), and the resulting composites were characterized. More recently, Park et al. ${ }^{38-40}$ utilized metallocene-based catalysts, such as $\mathrm{Cp}_{2} \mathrm{ZrCl}_{2}$ or $\mathrm{CpTiCl}_{3}$, adsorbed on the surface of MWNTs, for the synthesis of PE/ CNTs nanocomposite materials.

Herein, we report the synthesis of a titanium alkoxide catalyst, covalently attached to SWNTs and MWNTs, through a Diels-Alder cylcoaddition of a benzocyclobutene derivative. The kinetics of the L-lactide polymerization from the catalystfunctionalized MWNT surfaces was examined. In addition, the catalyst-functionalized SWNTs were used for the surface initiated titanium-mediated coordination polymerization of L-LA, $\mathrm{HIC}$, and $\epsilon$-CL.

\section{Experimental Section}

Materials. The MWNTs were provided by NanoLab (Watertown, MA) and were prepared through the chemical vapor deposition method, followed by purification with HF wash to remove residual catalyst. TEM analysis revealed that the diameters and lengths of MWNTs range from 30 to $55 \mathrm{~nm}$ and from $100 \mathrm{~nm}$ to $1 \mu \mathrm{m}$, respectively. It was found by TG analysis that the MWNTs contain a small quantity of iron or iron oxide particles as residue $(\sim 3$ wt $\%)$. The carboxylic groups, present on the MWNTs, were removed by annealing at $250^{\circ} \mathrm{C}$ for $2 \mathrm{~h}$ under air followed by heating to $450{ }^{\circ} \mathrm{C}$ for $2 \mathrm{~h}$ under $\mathrm{N}_{2}$. The SWNTs (length $500 \mathrm{~nm}$ to $1 \mu \mathrm{m}$ and diameter $1-1.2 \mathrm{~nm}, 86 \%$ purity, Carbon Nanotechnologies, TX) were prepared through the HiPco method and purified by multistage oxidation in moisture air at 225,325 , and $425^{\circ} \mathrm{C}$ for $2 \mathrm{~h}$, as reported elsewhere. ${ }^{41}$ After every stage of oxidation the SWNTs were collected and digested with $\mathrm{HCl}$ for $10 \mathrm{~h}$ and washed thoroughly to remove catalyst and amorphous carbon materials before subjecting into higher temperature oxidation. The carboxylic groups, present on the SWNTs, were removed by annealing at 450 ${ }^{\circ} \mathrm{C}$ for $2 \mathrm{~h}$ under $\mathrm{N}_{2}$. 4-Bromobenzocyclobutene (BCB-Br, 97\%) was kindly donated by Dow Chemical (USA). $\epsilon$-CL, L-LA, HIC, ethylene oxide (EO), tetradecane, triethylamine (TEA), toluene, methanol, and magnesium turnings $(99.99 \%)$ were purchased from
Aldrich, USA. Monomers and solvents (toluene, THF) were purified according to high vacuum technique standard procedures. ${ }^{42}$ Magnesium turnings were washed with distilled THF and dried under vacuum at $60^{\circ} \mathrm{C}$.

Synthesis of (1-Benzocyclobutene ethoxy)dichlorocyclopentadienyltitanium (BCB-EOTiCpCl $\mathbf{C l}_{2}$ ). 2-Hydroxyethyl benzocyclobutene $^{43}$ (BCB-EO) was used for the synthesis of the (BCBEOTiCpCl $\left.{ }_{2}\right)$ initiator (1) (Scheme 1). BCB-EO $(0.9 \mathrm{~g}, 6.0 \mathrm{mmol})$ was added to a dry $100 \mathrm{~mL}$ round-bottom flask containing $20 \mathrm{~mL}$ anhydrous toluene under argon. In a similar flask containing 20 $\mathrm{mL}$ anhydrous toluene, $\mathrm{CpTiCl}_{3}(1.5 \mathrm{~g}, 6.0 \mathrm{mmol})$ was added and stirred for a few min. The BCB-EO solution was transferred to a separatory funnel attached to the second flask and TEA $(1.0 \mathrm{~mL}$, $6.0 \mathrm{mmol}$ ) was added under argon. The BCB-EO solution was added dropwise over $15-20$ min to the flask containing the $\mathrm{CpTiCl}_{3}$. The separatory funnel was then removed, the flask was closed and attached to the vacuum line and then heated to $40^{\circ} \mathrm{C}$. The solution was left, under continuous stirring, overnight in order to evaporate the toluene and excess TEA. The yield of the final viscous liquid was almost quantitative, calculated from the flask weight difference.

Covalent Attachment of $\mathrm{BCB}-\mathrm{EOTiCpCl} \mathrm{C}_{2}$ on MWNT or SWNT Surface. The precursor initiator moieties were covalently attached to the MWNTs using Diels - Alder [4+2] cycloaddition $^{43}$ (Scheme 1). In a glass reactor, $40 \mathrm{mg}$ of annealed MWNTs or SWNTs and $8 \mathrm{~mL}$ of tetradecane were placed under argon. The solution was sonicated for 15 min to achieve maximum dispersion of the CNTs in the solvent. The mixture was then heated to 235 ${ }^{\circ} \mathrm{C}$ under stirring. Then, BCB-EOTiCpCl ${ }_{2}$ (1) $(1 \mathrm{~g}, 3.02 \mathrm{mmol})$ was added dropwise to the mixture with vigorous stirring under argon for $35 \mathrm{~min}$. After the addition, the reaction mixture was stirred for another $25 \mathrm{~min}$ at $235^{\circ} \mathrm{C}$ and then cooled to room temperature. The substituted benzocyclobutene grafted CNTs were collected through filtration using a $0.4 \mu \mathrm{m}$ Teflon membrane. To remove the excess ungrafted $\mathbf{1}$, the CNTs were washed repeatedly with toluene $(500 \mathrm{~mL})$. The procedure was monitored by thin liquid chromatography (TLC). The washing was stopped when the filtered solution contained no precursor initiator, as revealed by TLC. Finally, the grafted CNTs were dried under high vacuum, and stored at the glovebox.

Ring-Opening Polymerization of $\epsilon$-CL Using SWNT Grafted with Precursor Initiators. In a typical experiment, $18.0 \mathrm{mg}$ of the SWNT- $g$ - $\left(\mathrm{BCB}-\mathrm{EOTiCpCl}_{2}\right) 2$ (containing $11.7 \times 10^{-6} \mathrm{~mol}$, $21.5 \mathrm{wt} \% \mathrm{BCB} \mathrm{EOTiCpCl} \mathrm{C}_{2}$ ) initiator was added to a dry $50 \mathrm{~mL}$ Schlenk flask under argon, and I was dissolved in $2 \mathrm{~mL}$ of toluene. $\varepsilon$-CL $(1.5 \mathrm{~mL}, 13.6 \mathrm{mmol})$ was then introduced and the flask was placed in an oil bath at $120{ }^{\circ} \mathrm{C}$. The polymerization was allowed 


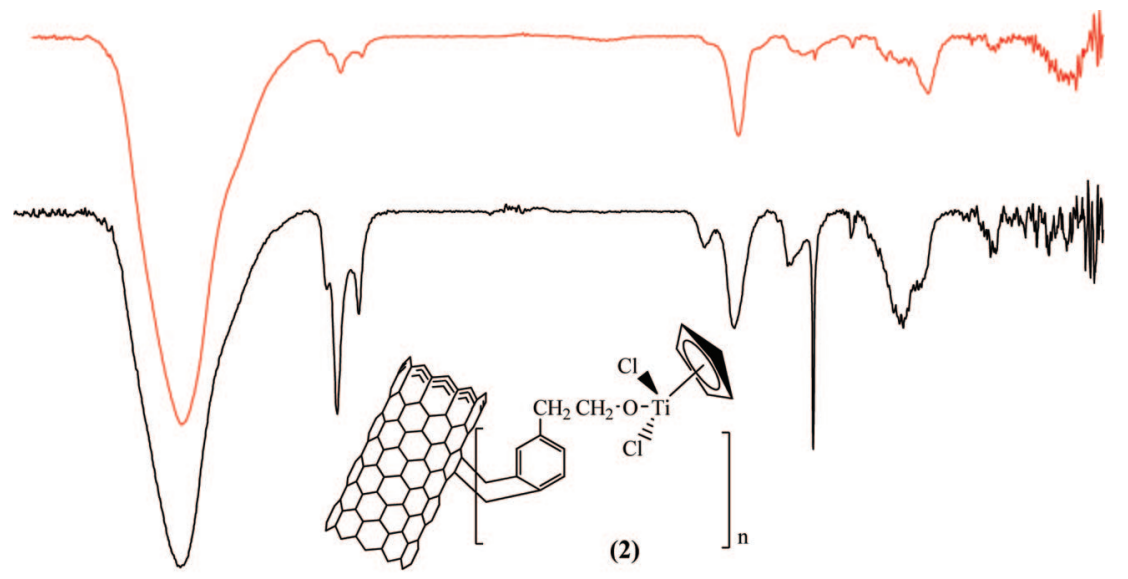

380036003400320030002800260024002200200018001600140012001000800600 Wavenumber $\left(\mathrm{cm}^{-1}\right)$

Figure 1. FTIR spectra of pristine SWNTs (red line), and functionalized SWNT- $g$-(BCB-EOTiCpCl ${ }_{2}$ ) (black line).

to proceed for $24 \mathrm{~h}$ and was terminated by addition of $5 \mathrm{~mL}$ of methanol, followed by dilution with toluene and filtration through a $0.4 \mu \mathrm{m}$ pore membrane. The residue was washed with toluene to remove excess monomer and any free polymer and was then dried under vacuum. A gray powder was isolated. The filtrate solution was concentrated and precipitated in methanol to collect a small quantity (traces) of free polymer.

Polymerization of HIC Using SWNT Grafted with Precursor Initiators. To a $50 \mathrm{~mL}$ Schlenk flask $26.3 \mathrm{mg}$ of 2 (containing $17 \times 10^{-6} \mathrm{~mol}, 21.5 \mathrm{wt} \% \mathrm{BCB}-\mathrm{EOTiCpCl} \mathrm{Cl}_{2}$ ) initiator was added, followed by addition of toluene $(1.0 \mathrm{~mL})$ and stirring. HIC (1.5 mL, $10.3 \mathrm{mmol})$ was then introduced, and the reaction was allowed to proceed at room temperature, under vigorous stirring. After $20 \mathrm{~h}$, the viscosity of the solution had not significantly increased. Termination was achieved by the addition of a $5 \%$ solution $(10 \mathrm{~mL})$ of $\mathrm{MeOH}$ in toluene, followed by dilution in toluene and filtration through $0.4 \mu \mathrm{m}$ pore membrane. The residue was washed with toluene to remove excess monomer and any free polymer and was then dried under vacuum.

Polymerization of L-LA Using MWNT and SWNT Grafted with Precursor Initiators. In a typical experiment, $25.0 \mathrm{mg}$ of $\mathbf{2}$ (containing $6.0 \times 10^{-6} \mathrm{~mol}, 8 \mathrm{wt} \% \mathrm{BCB}-\mathrm{EOTiCpCl} \mathrm{Cl}_{2}$ ) was added to a $50 \mathrm{~mL}$ Schlenk flask, and $\mathbf{I}$ was dissolved in $2 \mathrm{~mL}$ of toluene. L-LA $(1.0 \mathrm{~g}, 13.0 \mathrm{mmol})$ was then introduced, and the flask was placed in an oil bath at $130{ }^{\circ} \mathrm{C}$. The polymerization was allowed to proceed for up to $20 \mathrm{~h}$ and was terminated with methanol, followed by dilution in toluene and filtration through $0.4 \mu \mathrm{m}$ pore membrane. The residue was washed with toluene to remove excess monomer or any free polymer and dried under vacuum. A gray powder was isolated. The filtrate solution was concentrated and precipitated in methanol to collect small quantities of free polymer (when present).

Characterization. Thermogravimetric analysis was performed using a TA Instruments TGA Q500 model in air and nitrogen from 50 to $900{ }^{\circ} \mathrm{C}\left(10{ }^{\circ} \mathrm{C} / \mathrm{min}\right)$. Differential scanning calorimetry performed using TA instruments DSC Q 1000 model in nitrogen $\left(10^{\circ} \mathrm{C} / \mathrm{min}\right)$. FT-IR spectra were recorded using a Bio-Red WinIR Pro instrument with a resolution of $2 \mathrm{~cm}^{-1}$. $\mathrm{KBr}$ was used to prepare sample pellets. Raman spectra were acquired using samples in the solid state in the backscattering mode on the "microstage" of a Dilor XY Raman spectrometer (Instruments S.A., Inc., Edison, NJ). The $514.5 \mathrm{~nm}$ line of a Coherent Innova 200 argon ion laser was used for excitation. The laser output power $100 \mathrm{~mW}$ was attenuated by $\sim 86 \%$ when measured at the sample position. Spectra were recorded over the range $800-2000 \mathrm{~cm}^{-1}$ for $10-12$ acquisitions (with 20 s/acquisition) and averaged. Transmission electron microscopy (TEM) analysis was performed using a Hitachi H-800 TEM operating with an accelerating voltage of $100 \mathrm{kV}$. Samples were prepared by dropping a $\sim 5 \mu \mathrm{L}$ solution on a freshly glow discharged carbon film supported by a 400 mesh $\mathrm{Cu}$ grid. ${ }^{1} \mathrm{H}$ NMR was measured by using a $300 \mathrm{MHz}$ Bruker instrument using tetramethylsilane (TMS) as reference, and samples were made by dissolution in $\mathrm{CDCl}_{3}$. Circular dichroism spectra of PLLA grafted CNTs were taken in acetonitrile solutions using a Jasco J-815 CD Spectrometer.

\section{Results and Discussion}

Synthesis of Precursor Catalyst and Covalent Attachment onto CNTs. Substituted benzocyclobutenes (BCB) have been shown to react with the $\mathrm{sp}^{2}$ carbons of CNTs through a Diels-Alder reaction in a controllable way, giving a statistical distribution of $\mathrm{BCB}$ over the surface. ${ }^{15,43}$ For this reason, we decided to use 2-hydroxyethyl benzocyclobutene (BCB-EO) to create the precursor catalyst (Scheme 1).

4-Bromo benzocyclobutene was reacted with ethylene oxide to produce BCB-EO, which was subsequently reacted with $\mathrm{CpTiCl}_{3}$ to give the precursor catalyst $\mathbf{1}$. The final product was characterized by ${ }^{1} \mathrm{H}$ NMR spectroscopy (Figure S-2, Supporting Information). The characteristic signals at 2.8 and $4.6 \mathrm{ppm}$ correspond to the protons of the methylene groups, while the signals at 6.8-7.1 and 6.4-6.6 ppm correspond to the protons of the phenyl and cyclopentadiene rings, respectively.

The precursor catalyst $\mathbf{1}$ was covalently attached to both SWNTs and MWNTs through [4 + 2] cycloaddition (Scheme 1). A known amount of $\mathbf{1}$ was added dropwise to the pristine CNTs kept in tetradecane at $235^{\circ} \mathrm{C}$ to form [4+2] cycloadduct 2. 1 was kept at room temperature, prior to the addition to minimize dimerization of the benzocyclobutene. After the reaction, the functionalized CNTs were filtered and washed thoroughly with toluene to remove the remaining excess $\mathbf{1}$ and its oligomeric products. The IR spectra of the pristine and the catalyst-functionalized SWNTs are given in Figure 1. The presence of the $\mathrm{BCB}-\mathrm{EOTiCpCl} \mathrm{Cl}_{2}$ moiety on the SWNTs is confirmed by the appearance of the characteristic peaks of $\mathbf{1}$ such as $\mathrm{C}-\mathrm{H}$ stretching, aromatic ring bending and $\mathrm{C}-\mathrm{O}$ and Ti-O stretching which are clearly observed at 2925, 1500-1300, 1094, and 680-600 $\mathrm{cm}^{-1}$ respectively (Figure 1, black line) and do not exist in the IR spectra of the pristine CNTs (Figure 1 , red line). The bands at 3400 and $1600 \mathrm{~cm}^{-1}$ are attributed to the bending and scissoring vibrations respectively, of the trace water present in the $\mathrm{KBr}$ pellet used for measurements.

The Raman spectra of the pristine SWNTs (blue line) and the functionalized with $\mathbf{1}$ (red line) are provided in Figure 2. The characteristic tangential (G) and disorder (D) bands at 


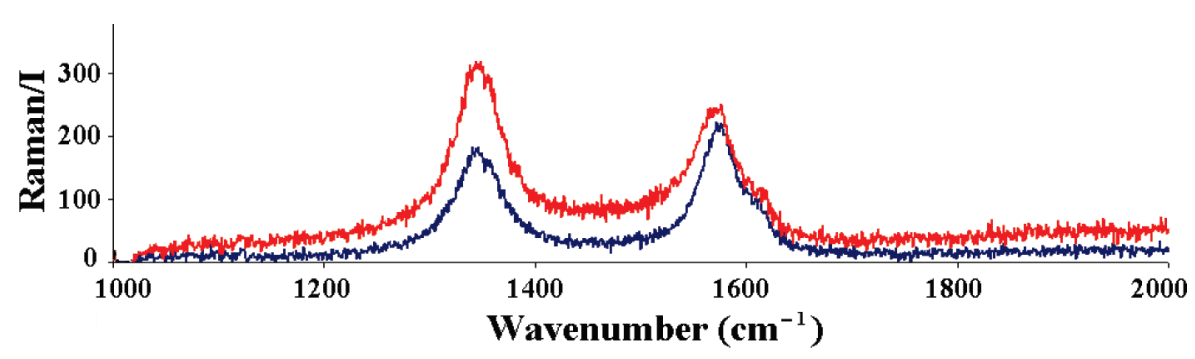

Figure 2. Raman spectra of pristine SWNTs (blue line), and functionalized SWNT- $g$-(BCB-EOTiCpCl 2 ) (red line).

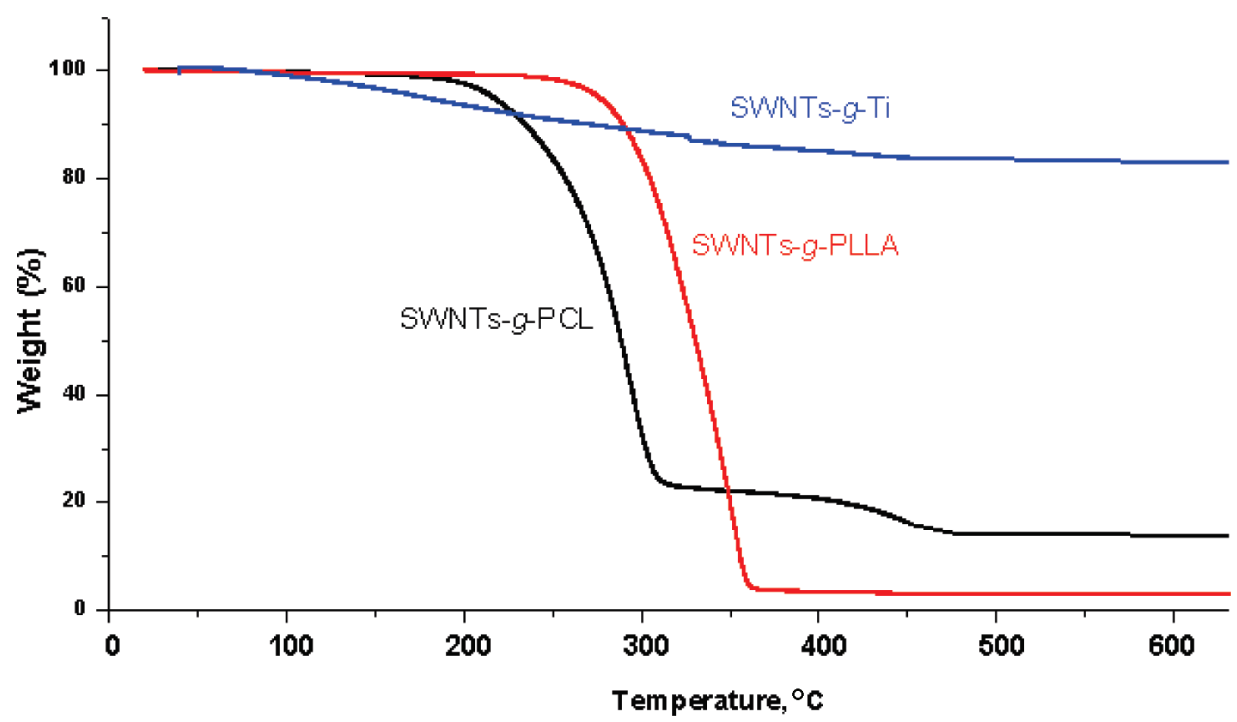

Figure 3. TGA of precursor initiator and polymers grafted from pristine SWNTs in $\mathrm{N}_{2}$ : (a) SWNT- $g$-(BCB-EOTiCpCl 2 ), 21.5 wt $\%$, (b) SWNT$g$-(PLLA), $94.9 \mathrm{wt} \%$, and (c) SWNT-g-(PCL), $78.2 \mathrm{wt} \%$.

Table 1. Molecular Characteristics of the Products Derived from the Surface-Initiated Polymerization of $\epsilon$-CL, L-LA, and HIC from Titanium-Catalyst-Functionalized SWNTs

\begin{tabular}{|c|c|c|c|c|c|c|c|}
\hline sample & $C_{\mathrm{SWNT}}^{a}\left(\times 10^{3}\right) \mathrm{mol}$ & $I_{\mathrm{SWNT}}^{b}\left(\times 10^{6}\right) \mathrm{mol}$ & $T_{\mathrm{d} \max }\left({ }^{\circ} \mathrm{C}\right)$ & {$[\mathrm{M}]_{\mathrm{mon}}(\mathrm{mol} / \mathrm{L})$} & $t(\mathrm{~h})$ & $\operatorname{convn}^{c}[\mathrm{M}]_{\mathrm{mon}}(\%)$ & $\operatorname{polymer}^{d}(\%)$ \\
\hline SWNT-g-PLLA & 1.9 & 14.9 & 350.4 & 5.5 & 20 & 52.3 & 91.7 \\
\hline SWNT- $g$-PLLA & 1.8 & 14.3 & 288.5 & 5.5 & 24 & 51.0 & 94.9 \\
\hline SWNT-g-PCL & 1.5 & 11.7 & 293.8 & 6.8 & 20 & 33.8 & 78.2 \\
\hline SWNT-g-PHIC & 2.2 & 17.0 & & 10.3 & 20 & 11.5 & \\
\hline
\end{tabular}

${ }^{a}$ mol of carbon in SWNTs. ${ }^{b}$ mol of $\mathbf{I}=[($ wt $\%$ of initiator by TGA/molecular weight of $\mathbf{I}$ fragment $\left.) / 100)\right] \times$ grams of SWNTs- $g$-(initiator $)_{n}$ taken for the reaction. I is the initiator fragment attached on the CNTs. ${ }^{c}$ Conversion of monomer, determined gravimetrically. ${ }^{d}$ The wt $\%$ of polymer on the surface of SWNTs as determined from TGA.

$\sim 1560$ and $1350 \mathrm{~cm}^{-1}$, respectively, are exhibited in both cases. However the peak ratio $I_{\mathrm{D}} / I_{\mathrm{G}}$ is slightly higher for the functionalized than the pristine SWNTs. The increase in the disorder (D) mode band corresponds to the conversion of the hybridization of the $\mathrm{C}$ atoms on the nanotubes from $\mathrm{sp}^{2}$ to $\mathrm{sp}^{3}$, which indicates covalent side-wall functionalization.

Thermogravimetric analysis (TGA) was used to determine the amount of precursor initiators grafted onto the SWNTs (Figure 3). The TGA thermogram of MWNT- $g$-(BCB-EOTiO$\mathrm{CpCl}_{2}$ ) showed an $8 \%$ weight loss between $250-450{ }^{\circ} \mathrm{C}$, corresponding to the decomposition of the precursor catalyst (1), whereas in the case of SWNTs- $g$ - $\left(\mathrm{BCB}-\mathrm{EOTiOCpCl} \mathrm{Cl}_{2}\right)$ it was $21.5 \%$. This difference in initiator wt $\%$ grafting, between the SWNTs and the MWNTs, was expected since SWNTS are more reactive, and since the reactions were performed under identical conditions.

Surface-Initiated Titanium-Mediated Coordination Polymerization of $\epsilon$-CL, HIC and L-LA from Catalyst-Functionalized SWNTs. The most definitive evidence for the successful synthesis and covalent attachment of the precursor-catalyst to the CNT surface was derived from its ability to initiate surface titanium-mediated coordination polymerization. The molecular characteristics of the polymers synthesized from the SWNT surface are presented in Table 1. As reported in the literature, attempts to graft PLLA on CNTs resulted in low grafting efficiency. Therefore, we decided to use similar polymerization times (up to $20 \mathrm{~h}$ ) to examine whether our grafting strategy has the same limitations. The polymerization proceeded up to $52 \%$ monomer conversion, and the PLLA-grafted SWNTs were obtained with a high degree of polymer grafting (>90\%). In the case of $\epsilon$-CL polymerization the monomer conversion was $34 \%$ and the polymer grafted on SWNTs was $78 \mathrm{wt} \%$ for $20 \mathrm{~h}$ polymerization time. Surprisingly, the HIC polymerization proceeded very slowly and there was only an $11 \%$ monomer conversion after $20 \mathrm{~h}$ polymerization time. The results of the polymerizations carried out using titanium alkoxide functionalized SWNTs are given in Table 1.

Thermograms of SWNT-g-(PLLA) and SWNT- $g$-(PCL) under $\mathrm{N}_{2}$ are provided in Figure 3. The weight losses between 200-300 and $260-360^{\circ} \mathrm{C}$ correspond to the decomposition of PCL and PLLA, respectively.

The ${ }^{1} \mathrm{H}$ NMR spectra of the polymerization products, (Figure 4), confirmed the presence of protons corresponding to the surface grown PCL, PHIC and PLLA. The characteristic chemical shifts of the ester methylene and methylene protons of PCL and PHIC are clearly seen at 4.1 and $3.6 \mathrm{ppm}$, 

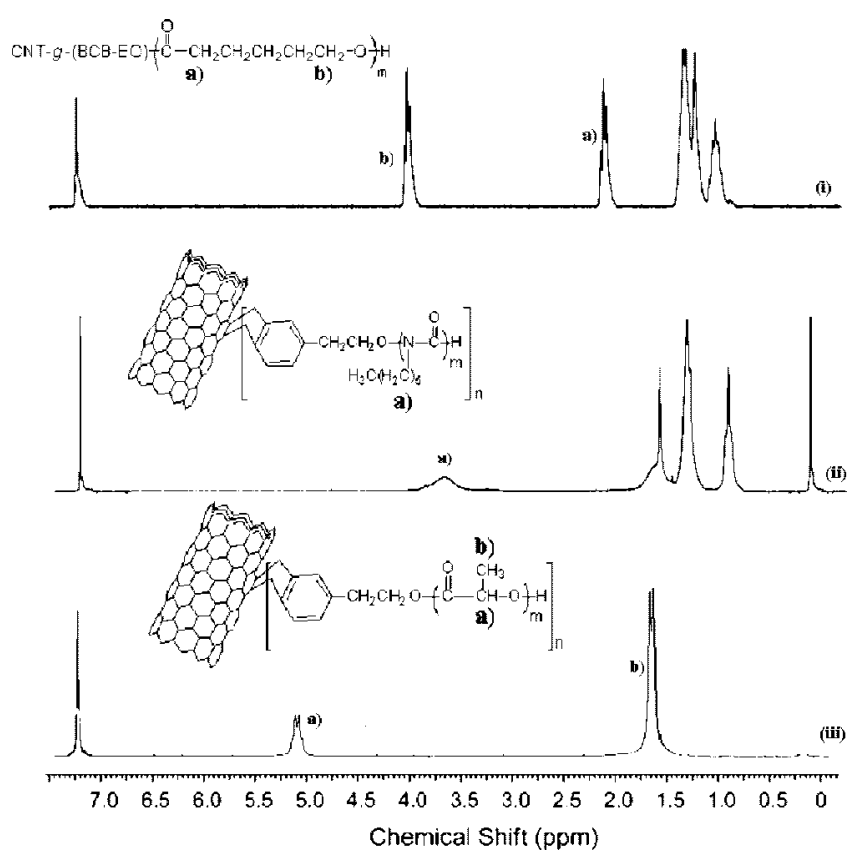

Figure 4. ${ }^{1} \mathrm{H}$ NMR spectra of (i) SWNT- $g$-(PCL) (sample 3, Table 1), (ii) SWNT-g-(PHIC) (sample 4, Table 1), and (iii) SWNT-g-(PLLA) (sample 2, Table 1), in $\mathrm{CDCl}_{3}$.

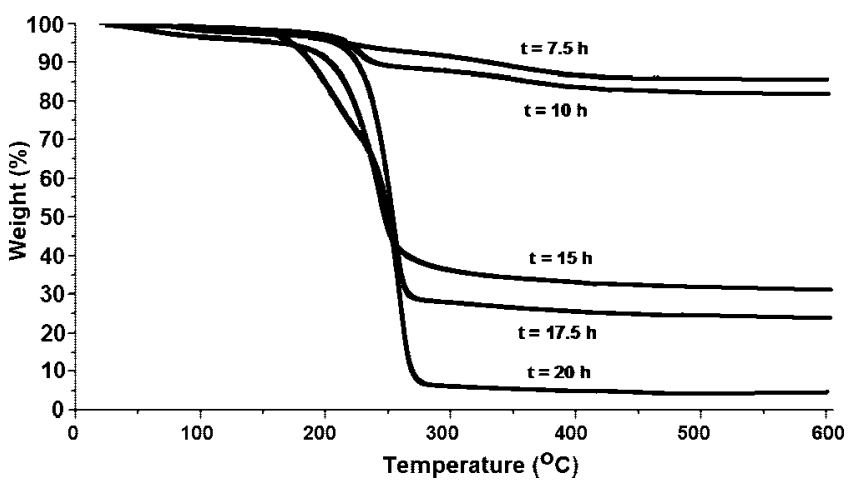

Figure 5. TGA of MWNTs- $-(\text { PLLA })_{n}$ at various reaction times in the surface-initiated titanium-mediated coordination polymerization of L-lactide from MWNT-g-(BCB-EOTiCpCl $)_{n}$ in toluene at $130{ }^{\circ} \mathrm{C}$.

respectively (Figures $4 \mathrm{i}$ and ii), while the characteristic chemical shifts at 5.2 and $1.6 \mathrm{ppm}$ are attributed to the $\mathrm{CH}$ and $\mathrm{CH}_{3}$ groups of the PLLA (Figure 4iii).

Surface-Initiated Titanium-Mediated Coordination Polymerization of L-LA from Catalyst-Functionalized MWNTs. Following the same procedure as for the SWNTs, the catalystfunctionalized MWNTs were used to initiate the polymerization of L-LA. The results of the surface initiated polymerization of L-LA for different durations $(7.5-20 \mathrm{~h}$ ) are presented in Table 2. The L-LA polymerization solution was initially heterogeneous, but with increasing reaction time the viscosity and homogeneity of the solution increased, thereby confirming the progress of the polymerization. The reaction was terminated by addition of a small amount of acidified methanol, followed by dilution with toluene. The MWNT-g-(PLLA) $)_{\mathrm{n}}$ samples were recovered by filtration using a $0.4 \mu \mathrm{M}$ Teflon membrane.

All grafting experiments were conducted with approximately similar monomer concentrations and amounts of MWNTs- $g$ $(\mathrm{BCB}-\mathrm{EOTiCpCl})_{n}\left(25 \mathrm{mg}\right.$, with $8 \%$ wt of $\mathrm{BCB}-\mathrm{EOTiCpCl} \mathrm{P}_{2}$ attached on MWNTs surface) Therefore, the increase in polymer grafting percentage (calculated from TGA) on the MWNTs surface was attributed to the length increase of the PLLA chains. The monomer conversion also increased with increasing reaction
Table 2. Surface-Initiated Titanium-Mediated Coordination Polymerization of L-LA from Catalyst-Functionalized MWNTs Using MWNTs- $g-\left(\mathrm{BCB}-E O T i C p C l_{2}\right)_{n}$, with 8 wt \% Grafted Catalyst in Toluene at $135{ }^{\circ} \mathrm{C}$

\begin{tabular}{ccccccc}
\hline run & $\begin{array}{c}C_{\mathrm{MWNT}}{ }^{a} \\
\left(\times 10^{3}\right) \mathrm{mol}\end{array}$ & $\begin{array}{c}I_{\mathrm{MWNT}}{ }^{b} \\
\left(\times 10^{6}\right) \mathrm{mol}\end{array}$ & $\begin{array}{c}{[\mathrm{L}-\mathrm{LA}]} \\
(\mathrm{mol} / \mathrm{L})\end{array}$ & $t(\mathrm{~h})$ & $\begin{array}{c}\text { convn }^{c} \\
{[\mathrm{~L}-\mathrm{LA}]}\end{array}(\%)$ & $\begin{array}{c}\text { PLLA }^{d} \\
(\%)\end{array}$ \\
\hline 1 & 2.1 & 6 & 5.6 & 7.5 & 3 & 13.8 \\
2 & 2.1 & 6 & 5.6 & 10.0 & 5 & 16.8 \\
3 & 2.1 & 6 & 5.6 & 12.5 & 9 & 39.8 \\
4 & 2.1 & 6 & 5.6 & 15.0 & 13 & 65.9 \\
5 & 2.1 & 6 & 5.6 & 17.5 & 27 & 74.5 \\
6 & 2.1 & 6 & 5.6 & 20.0 & 43 & 93.8
\end{tabular}

${ }^{a}$ mol of carbon in MWNTs. ${ }^{b}$ mol of $\mathbf{I}=[($ wt $\%$ of initiator by TGA/ molecular weight of $\mathbf{I}$ fragment)/100)] $\times$ grams of MWNTs- $g$-(initiator) ${ }_{n}$ taken for the reaction. ${ }^{c}$ Conversion of monomer, determined gravimetrically. ${ }^{d}$ The wt $\%$ of polymer on the surface of MWNTs as determined from TGA.

time (Table 2, run 1-6), leading to nanocomposites with very high percentage of PLLA (up to 94\%). These data provide direct evidence for controlled polymerization.

The reaction medium became very viscous and solidified at L-LA conversions higher than $40 \%(20 \mathrm{~h})$, indicating the formation of high molecular PLLA chains on the MWNT surface. However, in the cases of low L-LA conversions $(9 \%$ and lower), the MWNT-g-(PLLA) ${ }_{n}$ samples were not soluble enough for ${ }^{1} \mathrm{H}$ NMR characterization in solution. Direct evidence for the presence of surface grown PLLA was provided by the increase in weight (higher than $100 \%$ on the basis of MWNT$g$-(BCB-EOTiCpCl $)_{n}$ and from TGA. The TGA thermogram (Figure 5) corresponding to the product obtained after $15 \mathrm{~h}$ revealed a $65.9 \%$ weight loss (from $260-360^{\circ} \mathrm{C}$ ) (Table 2, run 4). At this point the monomer conversion was found to be $13 \%$, as calculated gravimetrically. A small portion of the MWNTs settled in the reactor during the polymerization (reaction times less than $15 \mathrm{~h}$ ) as a result of slow initiation, while those reactions carried out for longer times showed a complete dissolution of MWNTs during the polymerization. This suggests that the initiation of L-LA from the surface of MWNTs in a heterogeneous medium is very slow and has an induction period of about $15 \mathrm{~h}$.

The existence of an induction period was expected for such a surface-initiated polymerization. We have previously shown ${ }^{15}$ that for CNT surface initiated polymerization, an induction period occurs, even in the case of anionic polymerization of ethylene oxide. Furthermore, we have observed that the titaniummediated coordination polymerization of L-LA, using the same catalyst and solvent, in the absence of CNTs has an induction period of $1 \mathrm{~h} .{ }^{44}$ After this induction period, the solution medium became homogeneous and the viscosity of the reaction medium was substantially increased in less than $5 \mathrm{~h}$.

Metallocene-based catalysts, such as $\mathrm{Cp}_{2} \mathrm{ZrCl}_{2}$ or $\mathrm{CpTiCl}_{3}$, are known to adsorb onto the MWNT surface after mixing at room temperature for $6 \mathrm{~h} .^{38-40}$ This adsorption produces new precursor catalytic sites where the $\mathrm{Cp}$ rings interact with the CNTs through $\pi-\pi$ stacking. To investigate whether such a phenomenon took place here, a control experiment was carried out in which a known amount of a precursor catalyst $\mathrm{CpTiCl}_{2}\left(\mathrm{OCH}_{3} \mathrm{CH}_{2}\right)$, was placed in tetradecane in the presence of pristine MWNTs under stirring. The solution was heated, cooled, filtered and washed thoroughly following the same procedure and conditions as for $\mathrm{BCB}-\mathrm{EOTiCpCl} \mathrm{Cl}_{2}$-grafted MWNTs. Upon addition of the monomer and until the reaction was terminated (after $24 \mathrm{~h}$ ), no change in the reaction medium was observed. The viscosity and heterogeneity of the solution remained the same indicating no polymerization. Additional evidence was provided by the fact that the MWNTs, from the reaction mixture, showed no decomposition between 200-450 ${ }^{\circ} \mathrm{C}$ in TGA. These results confirm that the catalyst cannot be 

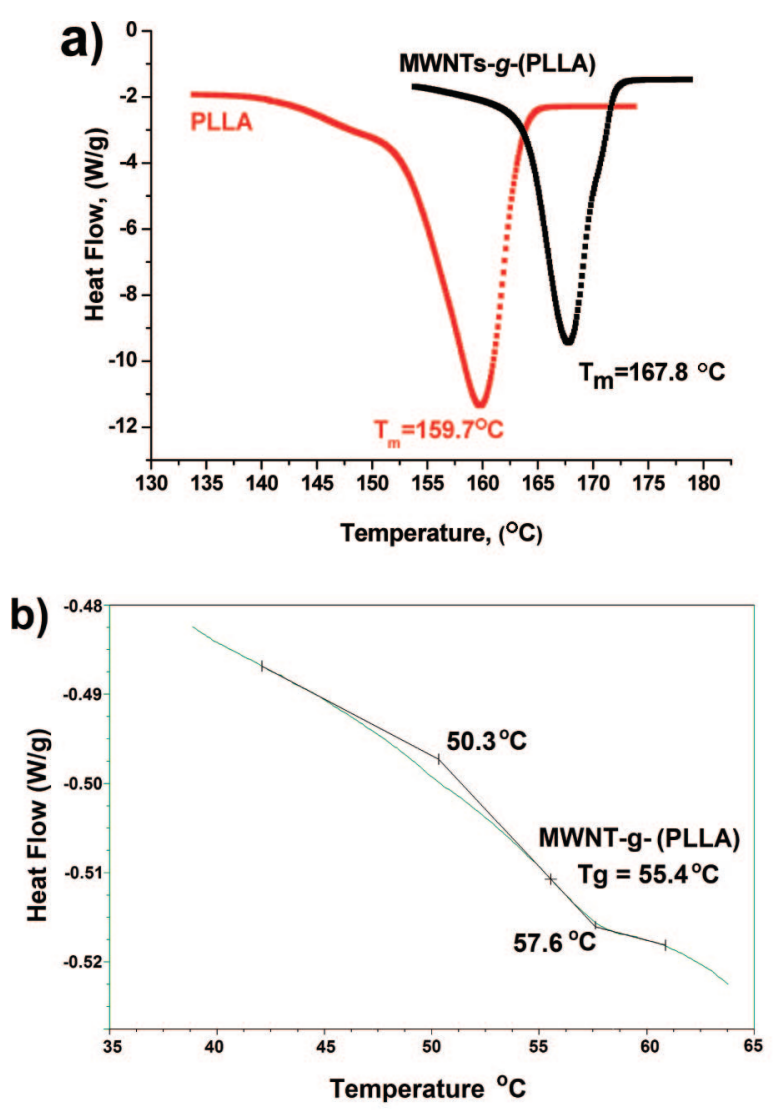
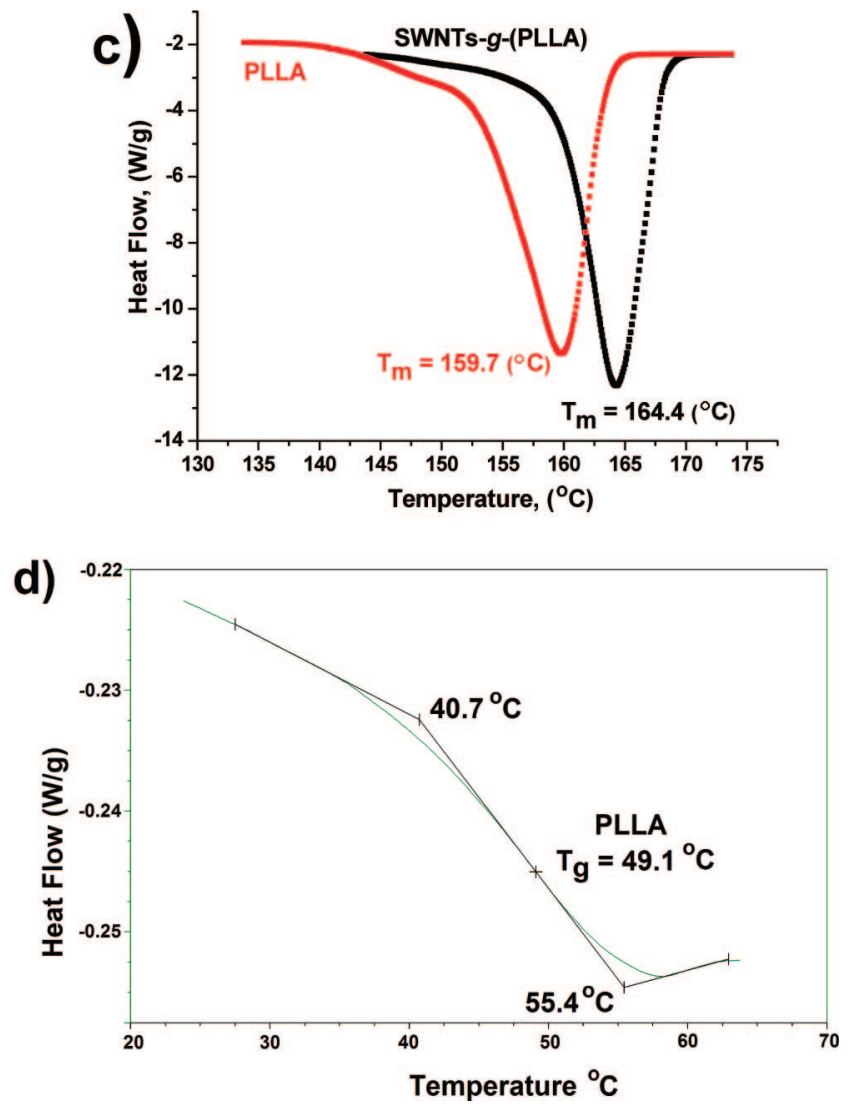

Figure 6. Differential scanning calorimetry thermograms of (a) MWNT- $g$-(PLLA) (run 5, Table 2) and bulk PLLA $\left(M_{\mathrm{n}}=10 \mathrm{~K}\right)$, (b) MWNT- $g$ $\left(\right.$ PLLA) $\left(\right.$ run 5, Table 2), (c) SWNT- $g$-(PLLA) (run 2, Table 1) and bulk PLLA $\left(M_{\mathrm{n}}=10 \mathrm{~K}\right)$, and (d) bulk PLLA $\left(M_{\mathrm{n}}=10 \mathrm{~K}\right)$.

adsorbed ( $\pi-\pi$ stacking) on the MWNT surface in the conditions used for our experiments and therefore polymerizations are initiated exclusively from the catalyst attached covalently.

Thermograms of MWNT-g-(PLLA) $n$ at different polymerization times, under $\mathrm{N}_{2}$, are given in Figure 5. A distinct weight loss in the temperature range of $200-370{ }^{\circ} \mathrm{C}$ corresponding to the degradation of grafted PLLA was obvious for all samples. The amount of PLLA attached on the MWNT surface increased with increasing reaction time.

DSC traces for bulk PLLA $\left(M_{\mathrm{n}}=10000, I=1.06\right)$ and MWNT-g-(PLLA) (run 5, Table 2) are given in Figure 6. The glass transition temperature $\left(T_{\mathrm{g}}\right)$ of the polymer grafted to MWNTs was found to be $55.4{ }^{\circ} \mathrm{C}$, whereas $49.1{ }^{\circ} \mathrm{C}$ for the bulk PLLA. The $6{ }^{\circ} \mathrm{C}$ increase was attributed to the decreased mobility of the PLLA chains due to their attachment to the MWNT surface. A similar increase was obvious in the melting temperature $\left(T_{\mathrm{m}}\right)$. Interestingly, the $T_{\mathrm{m}}$ of the PLLA increased, compared to the bulk PLLA, by 5 and $8{ }^{\circ} \mathrm{C}$, depending on its attachment to either SWNTs or MWNTs respectively. 45

The crystalline domains of PLLA have been extensively studied using X-ray diffraction, Raman and IR analyses revealing that they are composed of chains with a left-handed helix conformation ( $\alpha$-form), with 10 residues in three turns $\left(10_{3}\right.$ helix) similar to that of polypeptide $\alpha$-helices, such as poly(Lalanine $){ }^{46}$ At higher drawing temperatures another structure $(\beta$ structure) with an orthorhombic unit cell containing six chains with a 3 helical conformation has been observed. To date, circular dichroism spectroscopy has not been employed for the conformational analysis of poly(L-lactide), since PLLA gives a signal in the far UV and the majority of its solvents absorb strongly in this region.

We found that acetonitrile, a good solvent for PLLA, does not absorb in the far UV and was therefore used for CD measurements. In the case of neat PLLA, the positive maximum

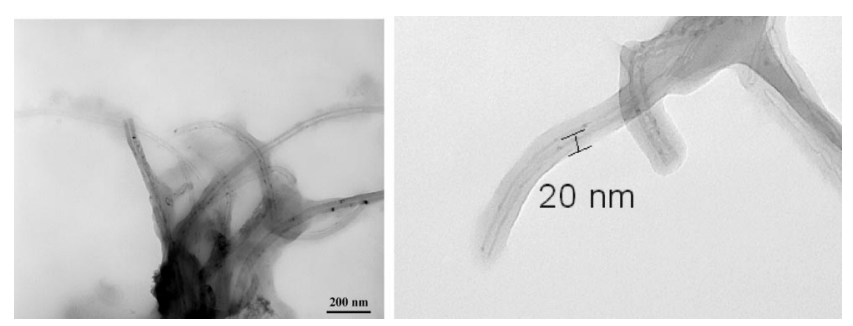

Figure 7. TEM images of MWNT-g-(PLLA) (run 6, Table 2).

centered at around $210 \mathrm{~nm}$, assigned to the $\pi-\pi^{*}$ electron transition of the ester chromophore, verified the $\alpha$-helix conformation (Figure S-1, Supporting Information). The CD spectra of SWNTs- $g$-PLLA and MWNTS- $g$-PLLA in acetonitrile were recorded at different temperatures $\left(25-75^{\circ} \mathrm{C}\right)$. In all cases and independently of the temperature, the molecular weight or degree of grafting, the spectra were similar to each other and to PLLA homopolymer. However, the intensity of the signal decreased with the increase in temperature, suggesting a gradual decrease of helicity with increasing temperature.

A dilute solution of MWNTs- $g$-(PLLA) $)_{n}$ in toluene was dropcoated onto a carbon grid and the thin film was visualized with TEM. An organic layer up to $23 \mathrm{~nm}$ thickness on the surface of MWNTs-g-(PLLA) $)_{n}$ was identified (Figure 7). It can also be concluded from the TEM images that the large CNT bundles are separated after being covered with the polymer layer due to the repulsion between the different polymer chains.

\section{Conclusions}

The synthesis and covalent attachment of BCB-EOTiCpCl catalyst through a Diels-Alder cycloaddition reaction on the surface of CNTs was achieved. This surface-grafted catalyst can 
efficiently promote the polymerization of L-LA and $\epsilon$-CL from single (SWNTs) and multiwalled carbon nanotubes (MWNTs). The initiation of L-LA from MWNTs was slow and characterized by an induction period of about $15 \mathrm{~h}$, due to the heterogeneous nature of the polymerization. After this induction period, the solution medium became homogeneous and the reaction proceeded rapidly. This surface-grafted catalyst was also employed for the polymerization of HIC. In contrast to the other two monomers, very low conversions were obtained. Differential scanning calorimetry proved that the glass transition and melting temperatures of the PLLA are affected by the presence of the CNTs, while PLLA $\alpha$-helix conformation remains the same, as revealed by the circular dichroism spectra.

Acknowledgment. The financial support of the Ministry of Education through the Operational Program and Initial Educational Vocational Training on "Polymer Science and its Applications" and the Research Committee of the University of Athens is greatly appreciated. The work at ORNL was supported by the U.S. Department of Energy, Basic Energy Sciences, Division of Materials Science and Engineering (DE-AC05-00OR22725) and through a user project to J.M. at the Center for Nanophase Materials Sciences. We thank Daniela Let for her help with CD experiments.

Supporting Information Available: Figure S-1, CD spectra of PLLA, SWNTs- $g$-(PLLA), and MWNTs- $g$-(PLLA) and Figure $\mathrm{S}-2,{ }^{1} \mathrm{H}$ NMR spectrum of BCB-EOTiCpCl ${ }_{2}$ in $\mathrm{CDCl}_{3}$. This material is available free of charge via the Internet at http://pubs.acs.org.

\section{References and Notes}

(1) Choi, W. B.; Chung, D. S.; Kang, J. H.; Kim, H. Y.; Jin, Y. W.; Han, I. T.; Lee, Y. H.; Jung, J. E.; Lee, N. S.; Park, G. S.; Kim, J. M. Appl. Phys. Lett. 1999, 75, 3129.

(2) Dai, H. J. Acc. Chem. Res. 2002, 35, 1035.

(3) Lefebvre, J.; Antonov, R. D.; Radosavljevi, M.; Lynch, J. F.; Llaguno, M.; Johnson, A. T. Carbon 2000, 38, 1745.

(4) Yu, M.-F.; Files, B. S.; Arepalli, S.; Ruoff, R. S. Phys. ReV. Lett. 2000, 84, 5552.

(5) Hamon, M. A.; Hu, H.; Bhowmik, P.; Niyogi, S.; Zhao, B.; Itkis, M. E.; Haddon, R. C. Chem. Phys. Lett. 2001, 347, 8.

(6) Baskaran, D.; Dunlap, J. R.; Mays, J. W.; Bratcher, M. S. Macromol. Rapid Commun. 2005, 26, 481.

(7) Baskaran, D.; Mays, J. W.; Bratcher, M. S. Polymer 2005, 46, 5050

(8) (a) Baskaran, D.; Mays, J. W.; Bratcher, M. S. Chem. Mater. 2005, 17, 3389. (b) Baskaran, D.; Sakellariou, G.; Mays, J. W.; Bratcher, M. S. J. Nanosci. Nanotechnol. 2007, 7, 1560.

(9) Baskaran, D.; Mays, J. W.; Bratcher, M. S. Angew. Chem., Int. Ed. 2004, 43, 2138

(10) Kong, H.; Gao, C.; Yan, D. J. Am. Chem. Soc. 2004, 126, 412.

(11) Xu, Y.; Gao, C.; Kong, H.; Yan, D.; Jin, Y. Z.; Watts, P. C. P. Macromolecules 2004, 3, 7-8846.

(12) Yao, Z.; Braidy, N.; Botton, G. A.; Adronov, A. J. Am. Chem. Soc. 2003, 125, 16015.

(13) Baskaran, D. Carbon: Nanotubes. In Encyclopedia of Inorganic Chemistry, 2nd ed.; King, B., Ed.; John Wiley \& Sons, Ltd.: Chichester, U.K., 2005; Vol. II, pp 730.

(14) Tasis, D.; Tagmatarchis, N.; Bianco, A.; Prato, M. Chem. Rev. 2006, $106,1105$.

(15) Sakellariou, G.; Ji, H.; Mays, J. W.; Baskaran, D. Chem. Mater. 2008, $20,6217$.

(16) Viswanathan, G.; Chakrapani, N.; Yang, H.; Wei, B.; Chung, H.; Cho, K.; Ryu, C. Y.; Ajayan, P. M. J. Am. Chem. Soc. 2003, 125, 9258.
(17) Xu, G.; Wang, Y.; Pang, W.; Wu, W. T.; Zhu, Q.; Wang, P. Polym. Int. 2007, 56, 847.

(18) Qin, S.; Qin, D.; Ford, W. T.; Herrera, J. E.; Resasco, D. E. Macromolecules 2004, 37, 9963.

(19) Zhao, X. D.; Fan, X. H.; Chen, X. F.; Chai, C. P.; Zhou, Q. F. J. Polym. Sci., Part A: Polym. Chem. 2006, 44, 4656.

(20) Zeng, H.; Gao, C.; Yan, D. Adv. Funct. Mater. 2006, 16, 812.

(21) Buffa, F.; Hu, H.; Resasco, D. E. Macromolecules 2005, 38, 8258.

(22) Liu, Y.; Adronov, A. Macromolecules 2004, 37, 4755.

(23) Gómez, F. J.; Chen, R. J.; Wang, D.; Waymouth, R. M.; Dai, H. Chem. Commun. 2003, 190.

(24) (a) Brode, G. L.; Koleske, J. V. J. Macromol. Sci., Pure Appl. Chem. 1972, A6, 1109. (b) Ikada, Y.; Tsuji, H. Macromol. Rapid Commun. 2000, 21, 117. (c) Perrin, D. E.; English, J. P. Handbook of Biodegradable Polymers; Domb, A. J., Kost, J., Wisemann, D. M., Eds.; Harwood Academic Publishers: Amsterdam, 1997; p 63. (d) Albertsson, A. C.; Varma, I. K. Biomacromolecules 2003, 4, 1466.

(25) (a) Uhrich, K. E.; et al. Chem. Rev. 1999, 99, 3181. (b) Dobrzynski, P.; Kasperczyk, J.; Bero, M. Macromolecules 1999, 32, 4735.

(26) Yang, L. P.; Pan, C. Y. Macromol. Chem. Phys. 2008, $209,783$.

(27) Olalde, B.; Aizpurua, J. M.; Garcia, A.; Bustero, I.; Obieta, I.; Jurado, J. M. J. Phys. Chem. C. 2008, 112, 10663.

(28) Chen, G. X.; Kim, H. S.; Park, B. H.; Yoon, J. S. Macromol. Chem. Phys. 2007, 208, 389.

(29) Song, W.; Zheng, Z.; Weiliang, T.; Wang, X. Polymer 2007, 48, 3658.

(30) (a) Patten, T. E.; Novak, B. M. J. Am. Chem. Soc. 1996, 118, 1906. (b) Patten, T. E.; Novak, B. M. J. Am. Chem. Soc. 1991, 113, 5065. (c) Patten, T. E.; Novak, B. M. Macromolecules 1993, 26, 436. (d) Hoff, S. M.; Novak, B. M. Macromolecules 1993, 26, 4067. (e) Hoff, S. M.; Novak, B. M. Macromolecules 2001, 34, 3849.

(31) Scollard, J. D.; McConville, D. H. J. Am. Chem. Soc. 1996, 118, 10008.

(32) Porri, L.; Giarrusso, A.; Ricci, G. Prog. Polym. Sci. 1991, 16, 405.

(33) Ricci, G.; Porri, L.; Giarrusso, A. Macromol. Symp. 1995, 89, 383.

(34) (a) Maxein, G.; Mayer, S.; Zentel, R. Macromolecules 1999, 32, 5747. (b) Ishizu, K.; Hatoyama, N.; Uchida, S. J. Polym. Sci., Part A: Polym. Chem. 2007, 45, 4037. (c) Mourmouris, S.; Kostakis, K.; Pitsikalis, M.; Hadjichristidis, N. J. Polym. Sci., Part A: Polym. Chem. 2005, 43,6503 .

(35) (a) Touris, A.; Kostakis, K.; Mourmouris, S.; Kotzabasakis, B.; Pitsikalis, M.; Hadjichristidis, Macromolecules 2008, 41, 2426. (b) Okuda, J.; Rushkin, I. L. Macromolecules 1993, 26, 5530.

(36) (a) Rodriguez-Delgado, A.; Marriot, W. R.; Chen, E. Y.-X. Macromolecules 2004, 37, 3092. (b) Chen, E. Y.-X. J. Polym. Sci., Part A: Polym. Chem. 2004, 42, 3395.

(37) Tong, X.; Liu, C.; Cheng, H. M.; Zhao, H.; Yang, F.; Zhang, X. J. Appl. Polym. Sci. 2004, 92, 3697.

(38) Park, S.; Yoon, S.; Lee, K. B.; Kim, D. J.; Jung, H. Y.; Do, Y.; Paik, H.; Choi, I. Macromol. Rapid Commun. 2006, 27, 47.

(39) Park, S.; Choi, I. Adv. Mater. 2008, 20, 1.

(40) Park, S.; Yoon, S. W.; Choi, H.; Lee, J.; Cho, W.; Kim, J.; Park, H.; Yun, W.; Do, Y.; Choi, I. Chem. Mater. 2008, 20, 4588.

(41) (a) Baskaran, D.; Mays, J. W.; Zhang, P.; Bratcher, M. S. J. Am. Chem. Soc. 2005, 127, 6916. (b) Chiang, I. W.; Brinson, B. E.; Huang, A. Y.; Willis, P. A.; Bronikowski, M. J.; Margrave, J. L.; Smalley, R. E.; Huage, R. H. J. Phys. Chem. B 2001, 105, 8297.

(42) (a) Hadjichristidis, N.; Iatrou, H.; Pispas, S.; Pitsikalis, M. J. Polym. Sci., Part A: Polym. Chem. 2000, 38, 3211. (b) Uhrig, D.; Mays, J. W. J. Polym. Sci., Part A: Polym. Chem. 2005, 43, 6179.

(43) Sakellariou, G.; Ji, H.; Mays, J. W.; Hadjichristidis, N.; Baskaran, D. Chem. Mater. 2007, 19, 6370.

(44) Petzetakis, N.; Let, D.; Pitsikalis, M.; Hadjichristidis, N. To be submitted.

(45) Trujillo, M.; Arnal, M. L.; Muller, A. J.; Laredo, E.; Bredeau, S.; Bonduel, D.; Dubois, P. Macromolecules 2007, 40, 6268.

(46) Kister, G.; Cassanas, G.; Vert, M.; Pauvert, B.; Tbol, A. J. Raman Spectrosc. 1995, 26, 307.

MA8027479 